accompanied by the secretion of AMF or other similar molecules by the tumour cells in large enough quantities to enable migration to take place. If this turns out to be the case, therapeutic possibilities open up, particularly that of inactivating AMF or its putative receptor by antibody blockade.

The relationship of MSF to familial breast cancer (and possibly other tumours) is also intriguing. How a dysfunction of fibroblast motility affects the breast epithelium is unclear: MSF may act primarily on hyaluronic acid synthesis and its effect on fibroblast migration may be a secondary consequence of changes in matrix organization. These changes in the extracellular matrix would then in turn affect breast epithelium metabolism. If a direct role for MSF is found in the aetiology of the disease, then ways to inhibit its action in vivo can be sought. Skin fibroblasts from about half the first-degree kin of people with familial breast cancer also show the fetal pattern of migration in collagen gels and may therefore secrete
MSF. Thus, screening relatives of people with breast cancer for MSF secretion by their skin fibroblasts may be a way to establish whether they are at risk.

Whether scatter factor is involved in the development of tumours also remains to be determined. This factor is secreted by various transformed fibroblast lines and a human teratocarcinoma line, so it is possible that it may allow fibrosarcomas to penetrate epithelia. There is no evidence yet that epithelial tumour lines make scatter factor: some of these lines are not responsive to the factor, but others retain sensitivity to it, so there may be complex interactions between epithelial tumours and the surrounding connective tissue. In addition, either one or more motility factor(s), or an as yet unknown factor, may mediate the tissue migration and remodelling which accompanies wound healing, and may well be of therapeutic value.

R.M. Warn and P. Dowrick are in the School of Biology, University of East Anglia, Norwich NR4 7TJ, UK

\title{
In situ differentiation in magma
}

\section{R. S. J. Sparks}

THE cooling, crystallization and chemical differentiation of magma in subterranean chambers is one of the principal causes of the great diversity of rocks in the Earth's crust. A central issue concerns how crystallization occurs in magma chambers and what the consequences are for magma evolution. The relative roles of crystal settling and in situ crystallization along the margins of a magma chamber have been widely debated. Hitherto, it has been assumed that the two mechanisms would be geochemically indistinguishable. But on page 199 of this issue ${ }^{1}$, Langmuir shows that this is not correct. He demonstrates that geochemical trends in magmas formed by in situ crystallization can be very different from those formed by equilibrium or perfect fractional crystallization. This is an important conceptual development as it offers the potential to distinguish different physical models in magma chambers. The new ideas also indicate that interpretations of igneous rock suites in terms of perfect fractional crystallization may sometimes be suspect.

Cooling of a magma chamber results in crystallization along the margins and in the interior which can be caused by convection allowing heat to be lost to the wallrocks. Crystals (whose composition differs from the melt's) nucleated at the margin can be swept into the interior to form a suspension or can remain at the margins growing in situ. Crystals in the interior can be sedimented out at the floor where further growth can occur in situ.
The relative importance of these possibilities is being hotly debated. For example Brandeis and Marsh $^{2}$ have argued that convection in magma chambers is weak so that most crystallization is confined to the margins. Others ${ }^{3,4}$ take the view that convection is important and that significant interior cooling, crystallization and differentiation results. There is also discussion on the relative importance of crystal settling and in situ crystallization ${ }^{5,6}$ because it has been recognized that when crystals grow, the evolved melt can convect away, allowing magma to differentiate - this is compositional convection.

In detail the margin of a magma chamber should consist of a mushy zone of crystals and melt separating entirely solidified rock from the interior magma. The thickness of this mushy zone could vary from negligible (the dimensions of a single layer of crystals) to hundreds of metres in the cumulate pile on the floor of a large chamber. Langmuir ${ }^{1}$ has examined the consequences of crystallization within this mushy zone and the exchange of interstitial melt with the main body of magma. Two extreme cases are worth consideration. First, there is no exchange so the trapped melt solidifies and the interstitial crystallization has no role in magma differentiation. Second, one can imagine a thin mushy zone where exchange is totally efficient so that any melt released by crystallization is immediately mixed into the interior. This case corresponds to perfect fractional crystallization. The interesting and more general case, however, is where the mush has significant depth and some part of the residual melt is exchanged with the interior. The principles of this situation have been investigated by Langmuir. These principles are simple but have far-reaching implications.

Consider as an illustration a crystal pile of plagioclase feldspar formed from a magma in which this is the only liquidus phase. Suppose at some depth in the mush the temperature has fallen to a value where olivine precipitates. The melt released from crystallization of olivine then exchanges with melt from the same chamber. Although the interior melt is in the plagioclase field, mixing with the more evolved melt will result in chemical trends which show the influence of olivine fractionation. Langmuir shows that profound departures from standard liquid lines of descent (evolution) are possible. The process also has implications for trace elements. Incompatible elements can be substantially enriched relative to major elements in comparison with enrichments generated by fractional crystallization. Langmuir cites several examples of igneous suites where these ideas explain features which were previously regarded as perplexing. For example, the enrichment of incompatible trace elements and the cryptic influence of clinopyroxene fractionation that is apparent in many mid-ocean-ridge basalts could be the consequence of in situ crystallization.

There is no need to dwell on the details of these results which are clearly explained in Langmuir's article'. There are, however, implications which can be taken further. The in situ model requires a physical process to exchange interstitial melt with the magma chamber. Compositional convection is the most plausible mechanism. The process also implicitly implies disequilibrium. If the more evolved, lower-temperature residual melts migrate through the mush into the interior then they must be heated up and would attempt to re-equilibrate with surrounding crystals. The residual melt must ascend fast enough to avoid significant thermal and chemical re-equilibration. These disequilibrium effects will further complicate the analysis of the mushy zone. The prospects are promising that the distinctive effects of in situ crystallization can be recognized from geochemical data and that these observations will help our understanding of the mechanisms operating in magma chambers.

R. S. J. Sparks is in the Department of Geology, University of Bristol, Bristol BS8 1RJ, UK.

1. Langmuir, C. H. Nature 340, 199-205 (1989).

2. Brandeis, G. \& Marsh, B. D. Nature 339, 613-616 (1989)

3. Martin, D., Griffiths, R. W. \& Campbell, I. H. Contrib. Mineral. Petrol. 96, 465-475 (1987)

4. Huppert, H. E. \& Sparks, R. S. J. A. Rev. Earth planet. Sci 12, 11-37 (1984)

5. Martin, D. Nature 327, 433 (1987)

6. Cox, K. G. \& Mitchell, C. Nature 333, 447-449 (1988) 